\title{
Oral contraception, parity, breast feeding, and severity of rheumatoid arthritis
}

\author{
C Jorgensen, M C Picot, C Bologna, J Sany
}

\begin{abstract}
Objective-To investigate the influence of breast feeding, use of the oral contraceptive pill (OCP), and parity on rheumatoid arthritis (RA).

Methods-One hundred and seventy six women with RA were compared with 145 control subjects; all had at least one child. RA patients were classified as having severe $(n=82)$ or mild disease $(n=89)$ according to clinical joint evaluation, radiological score, biological inflammation, and the presence of HLA-DR1 or -DR4 alleles.
\end{abstract}

Results-The mean age of RA patients was 58 years, and the mean age at the time of diagnosis of RA was 46 years. The mean time between onset of RA and the first birth was 23.6 (SD 3.8) years. The OCP user rates were $33 \%$ in the RA group and $47.6 \%$ in the control group $(p<0 \cdot 02)$. OCP use was related to the mother's year of birth. The relative risk for developing $R A$ was 0.598 (95\% confidence interval (CI) 0.33 to 1.1 ) in women who had used OCP for more than five years compared with those who had never used OCPs. In contrast, the age at which the first pregnancy occurred, the number of children breast fed, and the duration of breast feeding were comparable in $R A$ patients and healthy subjects. Among the RA patients, parity, duration of breast feeding, and the number of breast fed children were significantly increased in those with severe disease. Having more than three children increased the risk of developing severe disease $4 \cdot 8$-fold when adjusted for age and OCP use. Forty six percent of women with severe RA had a history of breast feeding duration greater than six months before disease onset, compared with $26 \%$ of patients with mild disease $(p<0.008)$. Having more than three breast fed children increased the risk of poor disease prognosis $3 \cdot 7$-fold. In contrast, OCP use had a protective role in the course of RA (44\% of RA patients with mild disease were OCP users, compared with $21 \cdot 7 \%$ of those with severe RA; $p<0.001$ ). Among those using OCP for more than five years, the relative risk of developing severe disease was $0.1(95 \% \mathrm{CI} 0.01$ to 0.6$)$, after adjustment for age, parity, and breast feeding.

Conclusion-Our results suggest that parity, and to a lesser extent breast feeding, before RA onset worsened RA prognosis, whereas OCP use had a protective role. Prolactin and oestrogen may have a role in these effects.

(Ann Rheum Dis 1996; 55: 94-98)

Partial or complete remission is obtained during pregancy in about $90 \%$ of patients with rheumatoid arthritis (RA). ${ }^{12}$ Sixty five percent of the women experience relapse of their symptoms within the first month and almost all patients suffer relapse by six months. ${ }^{34}$ Oka et al reported the onset of RA after pregnancy in $12.3 \%$ of women with RA, and pregnancy was suggested to be a risk factor for the development of RA. ${ }^{56}$ Pregnancy might influence the course of RA through lactation, and women who breast feed have a fivefold increase in the risk of developing RA within 12 months after pregnancy compared with controls. ${ }^{7}$ The same authors showed an eightfold increased risk for developing seropositive erosive disease in women who breast feed their children. In contrast, Hazes reported a decreased risk of RA in women who were pregnant before onset of the disease. ${ }^{8}$ The cause of relapse or development of RA in the postpartum period is unknown, but an action of the immunostimulant hormone prolactin is suspected. ${ }^{9}$ In the experimental model of collagen induced arthritis, bromocriptine (an antagonist of prolactin), given five days postpartum, suppressed the flare up observed in control animals. ${ }^{10}$ Moreover, in RA the pituitary prolactin response is upregulated and excessive secretion is observed after surgery. ${ }^{11}$

Use of the oral contraceptive pill (OCP) before the onset of RA symptoms has a protective effect against the disease, and reduces its severity. ${ }^{12}{ }^{13}$ Moreover, OCP use has been suggested to decrease the incidence of RA, but this remains to be clarified. ${ }^{14-16}$ The mechanisms of this phenomenon are unclear, and an immunosuppressive effect of oestrogen has been suggested. ${ }^{17}$ However, the lower incidence of pregancy and breast feeding in women who use oral contraception has not been taken into account.

In an attempt to analyse the effect of OCP use, parity, and breast feeding before disease onset on the relative risk of developing RA, the effect of past history of breast feeding on RA prognosis, and its relationship with OCP use and parity, we conducted a case control study of 176 women who had experienced at least one live birth. 


\section{Patients and methods}

PATIENTS

The study had a case control design. One hundred and seventy six female outpatients (age range 25-84 years) with at least one child were studied. The diagnosis of RA was made by one of the authors, according to criteria of the American Rheumatism Association, ${ }^{18}$ in the Immuno-Rheumatology Department, Gui de Chauliac Hospital, Montpellier France (the only referral clinic for inflammatory rheumatic disorders in the area). Mean disease duration at the time of the study was 10 years; mean age of RA patients was 58 (SD 12.3) years; age at the time of diagnosis of RA was 46 (14) years; mean time between RA onset and the first birth was $20 \cdot 8$ (15) years. A control group included 145 healthy women with at least one child who were involved in a systematic breast cancer screening programme in the geographic area of our rheumatology department. The age of this group was representative of that of the female population of the region. ${ }^{19}$

\section{QUESTIONNAIRE}

Information on parity, breast feeding (number of children and duration of breast feeding for each child), OCP use (including dates and duration of use), and the mean time between the first birth and the time of RA diagnosis was obtained by questionnaire accompanied by an explanatory letter mailed between February and April 1994. Two hundred and thirteen (94\%) of the 225 questionnaires sent to the patients were returned. One hundred and eighty three were suitable for analysis (86\%). We excluded six women in whom their first birth occurred after RA onset. In the control group ( $n=145$ ), the response rate was $74 \%$.

Table 1 Biological characteristics of the two groups of $R A$ patients

\begin{tabular}{lll}
\hline & $\begin{array}{l}\text { Mild } R A \\
(n=89)\end{array}$ & $\begin{array}{l}\text { Severe } R A \\
(n=82)\end{array}$ \\
\hline RF (\%) & 17 & 47 \\
Larsen score & $28 \cdot 5(25)$ & $66 \cdot 8(38)$ \\
ESR (mm/1st h) & $25 \cdot 3(19)$ & $52(32)$ \\
CRP (mg/l) & $15 \cdot 2(20)$ & $45(43)$ \\
HLA-DR1, HLA-DR4, or both (\%) & $47 \cdot 6$ & $83 \cdot 7$ \\
\hline
\end{tabular}

Values are \% or mean (SD).

$\mathrm{RF}=$ Rheumatoid factor; $\mathrm{ESR}=$ erythrocyte sedimentation rate; $\mathrm{CRP}=\mathrm{C}$ reactive protein.

Table 2 Demographic characteristics of $R A$ patients and controls

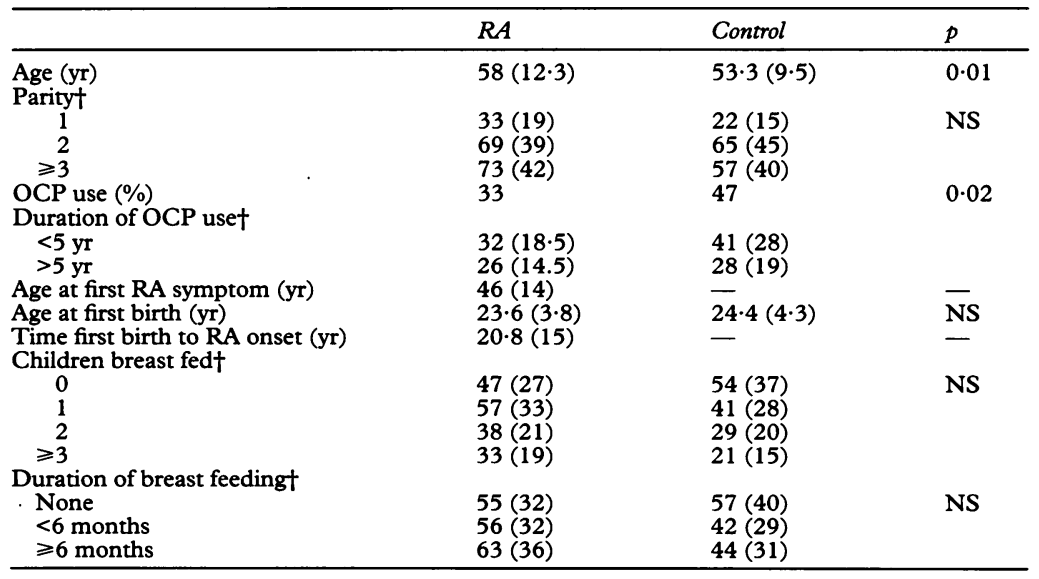

Values are mean $(\mathrm{SD})$ or + number $(\%)$. OCP = Oral contraceptive pill.
DATA COLLECTION

RA patients were examined within six months after they received the questionnaire. Joint tenderness, swelling, Ritchie index, ${ }^{20}$ and morning stiffness were recorded. Hand and wrist radiographs were scored according to Larsen's five point scale (maximum erosion score for wrist, metacarpophalangeal, and interphalangeal joints was 150). ${ }^{21}$ The presence of rheumatoid factor was determined by laser nephelometry, and a value greater than $40 \mathrm{IU}$ was considered positive. HLA-DR tissue typing was performed in RA patients, using the double fluorescence technique. Patients with RA were divided into two groups according to disease severity. Severe RA was defined by the association of four of the five following criteria: more than six persistently swollen joints and Ritchie index $>10$; Larsen's radiological score at the wrist $>30$; high titre rheumatoid factor; persistent biological inflammation at six month intervals (erythrocyte sedimentation rate $>30 \mathrm{~mm} / 1 \mathrm{st} \mathrm{h}$; $\mathrm{C}$ reactive protein $>15 \mathrm{mg} / \mathrm{l}$; association with HLA-DR1, -DR4, or both. In the absence of these criteria, RA was classified as mild disease $(52 \%$ of the RA patients had mild disease). Table 1 shows the characteristics of the two groups of RA patients.

STATISTICAL ANALYSIS

Demographic and other categorical variables were compared between the RA and control group and between severe RA and mild RA using the $\chi^{2}$ test or Fisher's exact test. The quantitative variables (age, age at RA onset, age at first birth) were compared using the Mann-Whitney test. The effects of parity, breast feeding, and OCP use were further examined by calculating crude odds ratios (OR) and $95 \%$ confidence intervals (CI). The logistic regression procedure was used to adjust the odds ratios for the following variables: age, OCP use, breast feeding, and parity. Possible confounding factors introduced as categorical variables included: duration of breast feeding (none or less than six months $v$ six months or more); parity (one, two, three, or more children); OCP use (none or less than five years $v$ five years or more); number of children breast fed (one, two, three, or more children). The software package SAS (version 6.08) was used for the analysis.

\section{Results}

COMPARISON OF RA AND CONTROLS

Table 2 compares the baseline characteristics for RA patients and controls. The control population was significantly younger. The OCP user rates in RA patients were lower compared with the control group ( $33 \% v 47.6 \% ; \mathrm{p}<0.02)$. However, OCP use was strongly associated with the mother's year of birth $(p<0.0001)$. The relative risk of developing RA among women who had used OCP for fewer than five years was less than in those who had never used OCPs $(O R=0.5,95 \%$ CI 0.29 to 0.87$)$, but was not statistically significant when adjusted for age at giving birth (table 3 ). 
Table 3 Estimated risk of developing $R A$ in women who used oral contraception (OCP) for fewer than five years or more than five years, compared with those who never used OCP

\begin{tabular}{llllll}
\hline OCP use & \multicolumn{2}{l}{ Number using } & & \multicolumn{2}{l}{ Odds ratio (95\% confidence interval) } \\
\cline { 2 - 3 } \cline { 6 - 7 } & $R A$ & Controls & & Crude & Adjusted $\dagger$ \\
\hline Never & 118 & 76 & & 1 & 1 \\
$<5$ years & 32 & 41 & & $0.50(0 \cdot 29$ to 0.87$)$ & $0.713(0.41$ to 1.23$)$ \\
$>5$ years & 26 & 28 & & $0.60(0.33$ to 1.1$)$ & $0.76(0.5$ to 1.14$)$ \\
\hline
\end{tabular}

tOdds ratios adjusted for age at birth, parity, and breast feeding, using logistic regression analysis.

We did not note any difference in parity, number of breast fed children, and total duration of breast feeding between RA patients and controls. We were not able to study the effect of nulliparity because all women selected had had at least one live birth.

Table 4 Oral contraceptive pill (OCP) use, parity, breast feeding, and RA severity

\begin{tabular}{|c|c|c|c|}
\hline & $\begin{array}{l}\text { Mild } \\
(n=89)\end{array}$ & $\begin{array}{l}\text { Severe } \\
(n=82)\end{array}$ & $p$ \\
\hline Age (yr) & $55 \cdot 6(12 \cdot 5)$ & $61(11 \cdot 0)$ & \\
\hline \multicolumn{4}{|l|}{ Duration of OCP use $†$} \\
\hline $\begin{array}{l}<5 \text { years } \\
>5 \text { years }\end{array}$ & $\begin{array}{l}16(18) \\
23(26)\end{array}$ & $\begin{array}{r}15(18) \\
2(2 \cdot 4)\end{array}$ & $\begin{array}{l}<0.002 \\
<0.001\end{array}$ \\
\hline OCP use (\%) & 44 & $21 \cdot 7$ & $<0.0001$ \\
\hline \multicolumn{4}{|l|}{ Parity† } \\
\hline 1 & $24(27)$ & $8(10)$ & \\
\hline 2 & $41(46)$ & $27(33)$ & \\
\hline$\geqslant 3$ & $24(27)$ & $46(57)$ & $<0.0001$ \\
\hline Age at first birth (yr) & $23 \cdot 6(3 \cdot 6)$ & $23 \cdot 5(4 \cdot 2)$ & NS \\
\hline Time first birth to RA onset (yr) & $22 \cdot 6(12 \cdot 4)$ & $24 \cdot 4(12 \cdot 8)$ & NS \\
\hline \multicolumn{4}{|l|}{ Duration of breast feeding } \\
\hline None & $31(36)$ & $24(29)$ & 0.02 \\
\hline 1-6 months & $33(38)$ & $20(24 \cdot 5)$ & \\
\hline$>6$ months & $23(26)$ & $38(46 \cdot 5)$ & \\
\hline \multicolumn{4}{|l|}{ Children breast fed } \\
\hline 0 & $26(29)$ & $21(26)$ & 0.008 \\
\hline 1 & $32(36)$ & $23(28)$ & \\
\hline 2 & $23(26)$ & $14(17)$ & \\
\hline$\geqslant 3$ & $8(9)$ & $24(29)$ & \\
\hline
\end{tabular}

Values are mean $(\mathrm{SD})$ or $\uparrow$ number $(\%)$.

Table 5 Estimated risk of mild $R A$ for women who used oral contraception (OCP) for fewer than five years or more than five years, compared with those who never used OCP

\begin{tabular}{llllll}
\hline OCP use & \multicolumn{2}{l}{ Number with $R A$} & & \multicolumn{2}{l}{ Odds ratio (95\% confidence interval) } \\
\cline { 2 - 3 } \cline { 6 - 7 } & Mild & Severe & & Crude & Adjusted $\dagger$ \\
\hline Never & 50 & 65 & & 1 & 1 \\
<5 years & 16 & 15 & & $0.7(0.35$ to $1 \cdot 6)$ & $0.9(0.2$ to $4 \cdot 0)$ \\
$>5$ years & 23 & 2 & & $0.07(0.01$ to 0.29$)$ & $0.1(0.01$ to 0.6$)$ \\
\hline
\end{tabular}

fOdds ratios adjusted for age at birth, parity, and breast feeding using logistic regression analysis.

Table 6 Estimated risk of severe $R A$ for women with two or three children compared with those with one child

\begin{tabular}{llllll}
\hline \multirow{2}{*}{ Parity } & \multicolumn{2}{l}{ Number with $R A$} & & \multicolumn{2}{l}{ Odds ratio (95\% confidence interval) } \\
\cline { 2 - 3 } \cline { 5 - 6 } & Mild & Severe & & Crude & Adjusted $\dagger$ \\
\hline 1 & 24 & 8 & & 1 & 1 \\
2 & 41 & 27 & & $1 \cdot 97(0 \cdot 77$ to $5 \cdot 04)$ & $2 \cdot 9(1 \cdot 0$ to $8 \cdot 3)$ \\
3 & 24 & 46 & & $5 \cdot 75(2 \cdot 24$ to $14 \cdot 7)$ & $4 \cdot 8(1.5$ to $15 \cdot 6)$ \\
\hline
\end{tabular}

fOdds ratio adjusted for age at birth, OCP use, and breast feeding using logistic regression analysis.

Table 7 Estimated risk of severe $R A$ for women who breast fed one or more child compared with those who never breast fed, and duration of breast feeding

\begin{tabular}{|c|c|c|c|c|}
\hline & \multicolumn{2}{|c|}{ Number with $R A$} & \multicolumn{2}{|c|}{ Odds ratio ( $95 \%$ confidence interval) } \\
\hline & Mild & Severe & Crude & Adjusted $\dagger$ \\
\hline \multicolumn{5}{|c|}{ Children breast fed } \\
\hline None & 26 & 21 & 1 & 1 \\
\hline 1 & 32 & 23 & $0.89(0.41$ to 1.95$)$ & $1.3(0.3$ to 0.6$)$ \\
\hline 2 & 23 & 14 & $1.3(0.5$ to 3.3$)$ & $3.6(0.6$ to 20.5$)$ \\
\hline 3 & 8 & 24 & $3.7(1.38$ to 9.95$)$ & $1.4(0.2$ to 10.0$)$ \\
\hline \multicolumn{5}{|c|}{ Duration of breast feeding } \\
\hline 0 & 31 & 24 & 1 & 1 \\
\hline 1-6 months & 33 & 20 & $0.9(0.38$ to 2.12$)$ & $1.65(0.71$ to 3.84$)$ \\
\hline$\geqslant 6$ months & 23 & 38 & $2 \cdot 13(1.02$ to 4.48$)$ & $0.96(0.41$ to 2.29$)$ \\
\hline
\end{tabular}

fOdds ratio adjusted for age at birth, OCP use, and parity using logistic regression analysis.
COMPARISON ACCORDING TO SEVERITY OF RA

$O C P$ use and $R A$ severity

Comparison of the two RA groups according to severity of the disease showed that OCP use before the onset of RA was significantly less in patients with severe RA (44\% OCP use in mild RA $v 21.7 \%$ in severe RA; $p<0.0001$ ) (table 4). Moreover, we observed a duration effect: $26 \%$ of RA patients with mild disease had used OCP for more than five years compared with only $2 \cdot 4 \%$ in the group of patients with severe disease. The relative risk of severe disease was $0 \cdot 7$-fold lower with OCP use of less than five years, and significantly lower with more than five years OCP use $(\mathrm{OR}=0.07,95 \% \mathrm{CI} 0.01$ to 0.3 ). However, the group with severe $\mathrm{RA}$ was significantly older than the group with mild RA (61 (11) years $v 55.6(12.5) ; \mathrm{p}<0.002)$. Table 5 shows that the protective effect of OCP remained after adjustment for age and parity $(\mathrm{OR}=0 \cdot 1$, $95 \%$ CI 0.01 to 0.6 ) with OCP use of more than five years.

Parity and $R A$ severity

Having had two children increased the risk of severe disease twofold, and with three or more children there was a $5 \cdot 75$-fold increased risk of poor prognosis of RA $(p<0.0001)$. These results remained significant when adjusted for age, OCP use, and breast feeding (table 6). Age at the first birth and the mean time between the first birth and onset of the disease did not modify the course of the disease in our study (table 4).

\section{Breast feeding and $R A$ severity}

The mean duration of breast feeding was increased in the RA group with severe disease, with an effect of duration: $46 \%$ of women with severe RA had a history of total breast feeding duration of more than six months, compared with $26 \%$ of those with mild RA $(p<0.02)$. A history of breast feeding of more than six months increased the risk of severe RA twofold, but this was not significant after adjustment for age, parity, and OCP use (table 7). In addition, the number of breast fed children was greater in women with severe RA compared with mild disease $(29 \%$ v $9 \%$ respectively; $p<0.008)$. The relative risk of severe RA with three or more breast fed children before RA onset was increased $1 \cdot 4$-fold (table 7 ).

\section{Discussion}

The results of the present study confirm the protective effect of OCP on the disease course of RA and suggest a decreased risk of developing RA among OCP users. Our results showed further that parity increased the risk of severe RA independently of OCP use and breast feeding before RA onset. In contrast, breast feeding was a confounding factor, but did not alter the course of the disease when adjusted for age or parity.

There were possible sources of bias in this study. The quality of the data collected could 
be questioned, as they were obtained through subject recall, and recall bias might have differed for controls and RA patients. However, random error in the exposure data, if present, would have led to dilution of any observed effect, and would not have modified the results. In addition, the two groups had similar questionnaire response rates. The control group selected from the breast cancer screening programme was chosen on the basis that it was representative of the local population. There are two concerns here: first, those that respond to an invitation to attend for screening may not be representative of the underlying population, and second, only $75 \%$ of those who attended for screening responded to the questionnaire for this study. It is thus very likely that the control groups studied might not have been representative in respect of their contraceptive, pregnancy, and breast feeding history. Unfortunately, we have no data on which to assess these issues and thus cannot exclude the possibility that the differences observed between the study and control groups may be explained, at least in part, by our method of selection of the controls. ${ }^{19}$ Breast feeding and OCP use might have been influenced by the social class of the women, but we had no information concerning this possible bias. However, random error in these data would be equally distributed in the two groups and is unlikely to have altered the results.

Information on OCP use and breast feeding was never a source of doubt, though dates and durations were sometimes questionable. In addition, all RA patients had definite disease, with a mean disease duration of 12 years, thus misclassification of the mean time between breast feeding and RA onset was unlikely.

There was clearly a marked cohort effect of year of birth on OCP use. Only three of the 51 RA patients older than 65 years had a history of OCP use. Women born before 1926 were unlikely to have used OCP. ${ }^{14}$ The apparent protective effect of OCP use on the risk of developing RA decreased after adjustment for year of birth.

Our results showed a protective effect of OCP on the course of RA (OR $=0 \cdot 1,95 \%$ CI 0 to $0 \cdot 6$ ) when adjusted for age, parity, and past lactation, and confirmed the findings of previous studies. ${ }^{12-14}$ However, the apparent decrease in the risk of developing RA was lower after adjustment for age, and Spector et al have shown that OCP use had no protective effect on the development of RA but prevented progression to severe disease. ${ }^{16}$ Sex hormone receptors are present on lymphocytes, and oestrogen has been shown to have an immunosuppressive effect on $\mathrm{T}$ cells. ${ }^{22}$ Moreover, postmenopausal oestrogen treatment has the same protective effect on the course of RA, suggesting that the effect of OCP is related to the immunosuppressive effect of sex hormones. ${ }^{23}$

OCP use is associated with a reduced number of children, and it has been suggested that the protective effect of OCP might be related to a decrease in parity. ${ }^{13}$ Our results suggest that having had more than three children did not modify the risk of developing $\mathrm{RA}$, but increased the risk of poor disease prognosis $4 \cdot 8$-fold. Multiparity was associated with more severe disease in past studies. ${ }^{1}$ We were not able to study the incidence of nulliparity on RA, because we selected women who had at least one child.

After a dramatic RA response during pregnancy, postpartum relapse occurs in more than $90 \%$ of patients within six months after they give birth. ${ }^{324}$ It seems that the timing of postpartum relapse is not correlated with lactation. ${ }^{2}$ In addition, pregnancy occurring before the disease onset decreases the risk of developing RA. ${ }^{8}$ However, the effect of breast feeding on the disease course has never been studied. Recently, a Norwegian study has shown a protective effect of lactation on $R A$ development, but this work was limited by the use of the death certificate as the source for the diagnosis. ${ }^{26}$ Our results suggested that breast feeding did not increase the risk of developing RA: duration of breast feeding was significantly related to severe RA but, when adjusted for age and parity, a history of lactation before disease onset did not alter the course of the disease. In contrast, an increased incidence of the relative risk of developing RA in the postpartum period has been reported, particularly for severe and erosive disease. ${ }^{7}$ One explanation for the possible effect of breast feeding on the development and course of RA might be a persistent increase in serum concentrations of prolactin during lactation. This hormone has an immunostimulant effect in vitro, and lymphocytes have been shown to express prolactin receptors. Moreover, in animal models of arthritis, arthritis improved after hypophysectomy or prolactin antagonist administration, and arthritis could be restored after prolactin injection. ${ }^{9}$ Postpartum flare up of collagen induced arthritis decreased after administration of bromocriptine. ${ }^{10}$ In RA, secretion of prolactin was shown to be upregulated, and excessively increased in case of stress. ${ }^{27}$ During pregnancy, immunosuppressive factors might explain the improvement in RA. ${ }^{28} 29$

Our results provide evidence that parity and previous OCP use modify the course of RA, but breast feeding seems to be a dependent effect. The underlying mechanisms are unknown, but our data should stimulate further research in the field of immunoendocrinology.

We wish to thank Dr J Cherif-Cheik (Institut Montpelliérain d'Imagerie Medico-biologique, 34196 Montpellier, Cedex 5) for her assistance.

1 Cecere F A, Perselin R H. The interaction of pregnancy and the rheumatic diseases. Clin Rheum Dis 1981; 7: 747-68.

Kaplan D, Diamond H. Rheumatoid arthritis and pregnancy. Clin Obstet Gynecol 1965; 8: 286-91.

3 Klipple G L, Cecere F A. Rheumatoid arthritis and pregnancy. Rheum Dis N Am 1989; 15: 213-39.

4 Ostensen M, Aune B, Husby G. Effect of pregnancy and hormonal changes on the activity of rheumatoid arthritis. Scand F Rheumatol 1983; 12: 69-72.

5 Oka $M$. Effect of pregnancy on the onset and course of rheumatoid arthritis. Ann Rheum Dis 1953; 73: 227-9.

6 Silman A J. Is pregnancy a risk factor in the causation of rheumatoid arthritis? Ann Rheum Dis 1986; 45: 1031-4.

7 Brennan P, Silman A. Breast feeding and the onset of rheumatoid arthritis. Arthritis Rheum 1994; 37: 808-13.

8 Hazes J M W, Dijizmans B A C, Vandenbroucke J P, DeVries R R P, Cats A. Pregnancy and the risk of RA. Arthritis Rheum 1990; 33: 1770-5. 
9 Berczi I, Nagy E, Asa S, Kovacs K. The inflence of pituitary hormones on adjuvant arthritis. Arthritis Rheum 1984; 27 682-8.

10 Whyte A, Williams R O. Bromocriptine suppresses postpartum exacerbation of collagen induced arthritis.

11 Chikanza I C, Panayi G S. Hypothalamic-pituitary mediated modulation of immune function: prolactin as a neuroimmune peptide. $B r \mathcal{F}$ Rheumatol 1991; 30: 203-7.

azes J M W, Vandenbroucke J P, Dijksman B A C, Cats A. Diminished incidence of severe rheumatoid arthritis associated with oral contraceptive use. Arthritis Rheum 1990; 33: 1462-5.

13 Hazes J M W, Dijkmans B A C, Vandenbroucke J P, Vries R R P, Cats A. Reduction of the risk of rheumatoid arthritis among women who take oral contraceptives. Arthritis Rheum 1990; 33: 173-9.

14 Spector T D, Roman E, Silman A J. The pill, parity, and rheumatoid arthritis. Arthritis Rheum 1990; 33: 782-9.

15 Linos A, Worthington J W, O'Fallon W N. Case control study of rheumatoid arthritis and prior use of oral study of rheumatoid arthritis and prior

16 Spector T D, Hochberg M C. The protective effect of the oral contraceptive pill on rheumatoid arthritis: an overview of the analytic epidemiological studies using metaanalysis. F Clin Epidemiol 1990; 43: 1221-30.

17 Jorgensen C, Sany J. Modulation of the immune response by the neuroendocrine axis in rheumatoid arthritis. Clin Exp Rheumatol 1994; 12: 435-41.

18 Arnett F C, Edworthy S M, Bloch D A, et al. The American Rheumatism Association 1987 revised criteria for the classification of rheumatoid arthritis. Arthritis Rheum 1988; 31: 315-24.

19 Seguret F, Daures J P, Guizard A V, et al. Herault breast cancer screening program: results after 30 months of the mobile French schedule. Eur $\mathcal{f}$ Cancer Prev 1995. In press.
20 Ritchie P M, Boyle J A, McInnes J M. Clinical studies with an articular index for the assessement of joint tenderness in patients with RA. $Q f$ Med 1969; 3: 393-406.

21 Larsen A, Dale K, Eek M. Radiographic evaluation of RA and related conditions by standard reference films. Acta Radiol Diag 1977; 18: 488-91.

22 Cutolo M, Accardo S, Villaggio B, et al. Presence of estrogen binding sites on macrophage like synoviocytes and $\mathrm{CD} 8+, \mathrm{CD} 29+, \mathrm{CD} 45 \mathrm{RO}+\mathrm{T}$ lymphocytes in normal and rheumatoid synovium. Arthritis Rheum 1993; 36: 1087-97.

23 Vandenbroucke J P, Witteman J C M, Valkenbourg $H$ A, Boersma J W, Cats A, Festen J J M. Noncontraceptive hormones and rheumatoid arthritis in perimenopausal and post menopausal women. $\neg A M A$ 1986; 255: and post

24 Felbo $M$, Snorrasson E. Pregnancy and the place of therapeutic abortion in rheumatoid arthritis. Acta Obstet Gynecol Scand 1961; 8: 116-26.

25 Oka M, Vainio U. Effect of pregnancy on prognosis and serology of rheumatoid arthritis. Acta Rheum Scand 1966; 12: 47-53.

26 Brun J G, Nilssen S, Kvales G. Breast feeding, other reproductive factors and rheumatoid arthritis. A prospective study. $B r \mathcal{F}$ Rheumatol $1995 ; 34: 542-6$.

27 Jorgensen C, Bressot N, Lefebre P, Bologna C, Suquet J, Sany J. Dysregulation of the pituitary adrenal axis and of the pituitary prolactin synthesis in rheumatoid arthritis. the pituitary prolactin synthesis in rheumatoid arthritis 28 Unger 1 Ratol 1995 . In press.

Unger A, Kay A, Griffin A J, Panayi G S. Disease activity and pregnancy associated $\alpha 2$ glycoprotein in rheumatoid arthritis during pregnancy. $B M \mathcal{F} 1983 ; 5$ : $750-2$.

29 Stanworth DR. A possible immunochemical explanation for pregnancy associated remissions in rheumatoid arthritis Ann Rheum Dis 1988; 47: 89-90. 